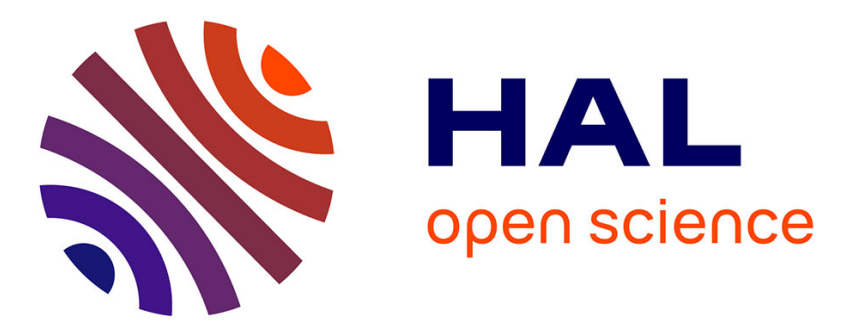

\title{
Dégradation et transformation d'un compost pétrolier apporté à des sols battants et effets sur leurs propriétés physiques
}

\author{
H. Mettauer, H. Bouslah, B. Guillet, S. Bruckert, Ch. Huck
}

\section{- To cite this version:}

H. Mettauer, H. Bouslah, B. Guillet, S. Bruckert, Ch. Huck. Dégradation et transformation d'un compost pétrolier apporté à des sols battants et effets sur leurs propriétés physiques. Agronomie, 1987, 7 (6), pp.391-399. hal-00885006

\section{HAL Id: hal-00885006 https://hal.science/hal-00885006}

Submitted on 1 Jan 1987

HAL is a multi-disciplinary open access archive for the deposit and dissemination of scientific research documents, whether they are published or not. The documents may come from teaching and research institutions in France or abroad, or from public or private research centers.
L'archive ouverte pluridisciplinaire HAL, est destinée au dépôt et à la diffusion de documents scientifiques de niveau recherche, publiés ou non, émanant des établissements d'enseignement et de recherche français ou étrangers, des laboratoires publics ou privés. 


\title{
Dégradation et transformation d'un compost pétrolier apporté à des sols battants et effets sur leurs propriétés physiques
}

\author{
H. METTAUER, H. BOUSLAH*, B. GUILLET* et S. BRUCKERT** \\ avec la collaboration technique de $\mathrm{Ch}$. $\mathrm{HUCK}^{*}$ \\ I.N.R.A., Station d'Agronomie, 28, rue de Herrlisheim, F 68021 Colmar \\ * Centre de Pédologie biologique, CNRS, BP 5, F 54501 Vandouvre-lès-Nancy \\ ** Pédologie, Université, 1, place Leclerc, F 25030 Besançon
}

La recherche réalisée visait à étudier le devenir d'un compost pétrolier après incorporation dans le sol et à apprécier sa valeur d'amendement. L'expérimentation a été effectuée au laboratoire sur 2 sols de texture limoneuse enrichis en compost selon une dose unique ( $40 \mathrm{t} / \mathrm{ha}$ ). Les échantillons maintenus à une humidite constante $(\mathrm{pF}=3)$ ont subi une période d'incubation de 9 mois.

La biodégradation du compost a été appréciée par l'évaluation de la quantité de carbone dégagé sous forme de $\mathrm{CO}_{2}$ et par la comparaison des activités ${ }^{14} \mathrm{C}$ naturelles en début et en fin d'expérience. Ces mesures ont été complétées par l'étude du fractionnement de la matière organique en vue d'estimer les quantités de carbone résiduel, extractible, humifié ou fixé à la matrice organo-minérale pré-existante.

Le taux de minéralisation du compost incorporé à une alluvion sablo-limoneuse a été de 5 p. 100 et de 10 p. 100 dans le cas d'un mélange avec un limon décarbonaté (lehm). L'incubation a également entrainé un transfert partiel du carbone de la fraction insoluble granulométriquement fine vers la fraction soluble dans le pyrophosphate de sodium.

Les effets d'amendement du compost pétrolier sont globalement faibles ; s'il améliore la stabilité structurale des sols en réduisant le degré de mouillabilité, il n'a pas d'effet significatif sur la sensibilité au tassement, le domaine de plasticité et la réserve utile en eau. La valorisation agricole d'un tel compost doit donc être envisagée avec prudence car sa toxicité n'est pas négligeable.

Mots clés additionnels : Biodégradation, activité ${ }^{14} \mathrm{C}$, fractionnement de la matière organique, amendement, propriétés physiques. properties.

The transformation of a fuel manure incorporated into soil was studied and its quality as a soil amendment was evaluated. The experiment was carried out under controlled conditions. The samples of fuel manure incorporated into two loamy soils were incubated for 9 months at constant humidity $(\mathrm{pF}=3)$. The biodegradation of the fuel manure was assessed by the amount of evolved $\mathrm{CO}_{2}$ and by comparison of ${ }^{14} \mathrm{C}$ natural activities at the beginning and the end of the experiment. These measurements were followed by analysis of the fractional distribution of the organic matter in order to estimate the quantities of residual, extractable and humified carbon or of carbon fixed to the pre-existing organic and mineral matrix. The mineralization rate of fuel manure incorporated into an alluvial silty soil was $5 \%$ and into a decarbonated loam $10 \%$. Incubation also induced a partial transfer of carbon from the insoluble fine fraction to the sodium pyrophosphate-soluble fraction. The effects of the fuel manure as an amendment were overall rather poor. The waste improved soil structural stability by reducing the wetting rate but its effects on sensitivity to compaction, plasticity index and water capacity were not significant. The agricultural use of such a fuel manure should be carefully considered because of its significant toxicity.

Additional key words : Biodegradation, natural ${ }^{14}$ C activity, amendment, fractionation of organic matter. 


\section{INTRODUCTION}

Les déchets d'origine industrielle posent de multiples problèmes quand il s'agit de s'en défaire, en décharge, sans nuire à l'environnement, ou quand on envisage leur utilisation à des fins agricoles. C'est le cas des composts pétroliers, produits fabriqués à partir de boues hydrocarburées de raffinerie de pétrole améliorées par compostage.

L'épandage sur le sol de compost pétrolier est une des voies d'élimination pratique des boues hydrocarburées connue sous le nom de "sludge farming ». A la longue, le compost pétrolier se biodégrade et se transforme en s'intégrant plus ou moins intimement aux constituants minéraux et organiques des sols.

Au plan de la recherche, qu'il s'agisse des expériences de laboratoire ou de terrain, c'est le suivi de la dégradation des composts pétroliers apportés aux sols qui a suscité le plus grand nombre de travaux, comme le reflète le dépouillement des références bibliographiques collectées en 1981 par l'atelier "Hydrocarbures dans les sols » du Ministère de l'Environnement. Dans une récente synthèse à l'Institut Français du Pétrole, GATELLIER (1983) fait état d'un taux moyen annuel de dégradation d'environ 50 p. 100, mais se demande si la « disparition " du compost correspond à une minéralisation effective ou bien à une dégradation en un produit résiduel non décelé par l'analyse. CARBONEL et al., 1981, posent la question du progrès de nos connaissances sur le devenir de la fraction résiduelle dont les composés polaires augmentent avec le temps et nouent des liens avec les constituants organo-minéraux des sols. C'est là un premier aspect du problème, abordé dans cette note.

Notre deuxième préoccupation a été de savoir si le compost pétrolier peut avoir un effet d'amendement organique notable. BouSLAH (1982) a en effet montré que, malgré une phytotoxicité relative, les composés pétroliers peuvent avoir un rôle positif en améliorant la stabilité structurale des sols battants. De tels produits pourraient remplacer avantageusement des substances plus onéreuses (résine, latex, émulsion de copolymères) proposées pour améliorer la stabilité structurale des sols.

Nos recherches ont donc consisté à suivre, au laboratoire, la dégradation d'un compost apporté selon une dose unique à 2 sols de texture limoneuse.

\section{MATÉRIEL ET MÉTHODES}

\section{A. Les matériaux initiaux : sols et compost pétrolier}

Les échantillons de sol proviennent de la plaine d'Alsace. Il s'agit :

- d'un horizon Ap limono-sableux prélevé dans un sol brun alluvial,

- d'un horizon Ap limoneux prélevé dans un sol brun lessivé de lehm (loess décarbonaté d'origine éolienne).

Nous les désignerons dans le texte par les termes d'alluvions et de lehm.

Le compost pétrolier provient de boues de raffinerie qui ont fait l'objet d'un compostage sur dalles drainantes chauffées en hiver. La raffinerie de REICHSTETT (Bas-Rhin) traite ainsi quelques $3000 \mathrm{~m}^{3}$ de boues par an. Les caractéristiques essentielles des sols et du compost pétrolier sont données dans le tableau 1. Il faut noter que les taux de carbone indiqués sont des moyennes accompagnées d'une erreur standard, qui résulte de l'analyse de 5 à 8 dosages de carbone, réalisés sur un même échantillon après quartage.

\section{B. Réalisation des mélanges sols-compost pétrolier}

Compte tenu du travail préliminaire de BousLAH (1982), réalisé avec diverses doses de compost, il a été décidé de simuler un apport unique au champ de $40 \mathrm{t} / \mathrm{ha}$ pour avoir un rapport Carbone du résidu/Carbone du sol plus élevé. En considérant un tonnage standard des horizons Ap de $3000 \mathrm{t} / \mathrm{ha}$, on obtient ainsi un rapport pondéral de 1/75.

Compte tenu de ce rapport et des teneurs respectives en carbone organique des sols et du compost, les mélanges présentent les teneurs en carbone indiquées dans le tableau 2. On en déduit que la part du carbone organique venant du compost pétrolier représente près de $1 / 4 \mathrm{du}$ carbone total dans le mélange alluvionscompost $(24,45 \pm 0,20 \mathrm{p}$. 100$)$ et près du $1 / 5$ pour le mélange lehm-compost $(19,40 \pm 0,20 \mathrm{p}$. 100).

\section{Protocole de mise en incubation}

Les mélanges ont été réalisés par retournement à la bétonnière. Après homogénéisation, des lots de deux

TABLEAU 1

Caractéristiques principales des sols et du compost pétrolier. Main characteristics of soils and of the fuel manure.

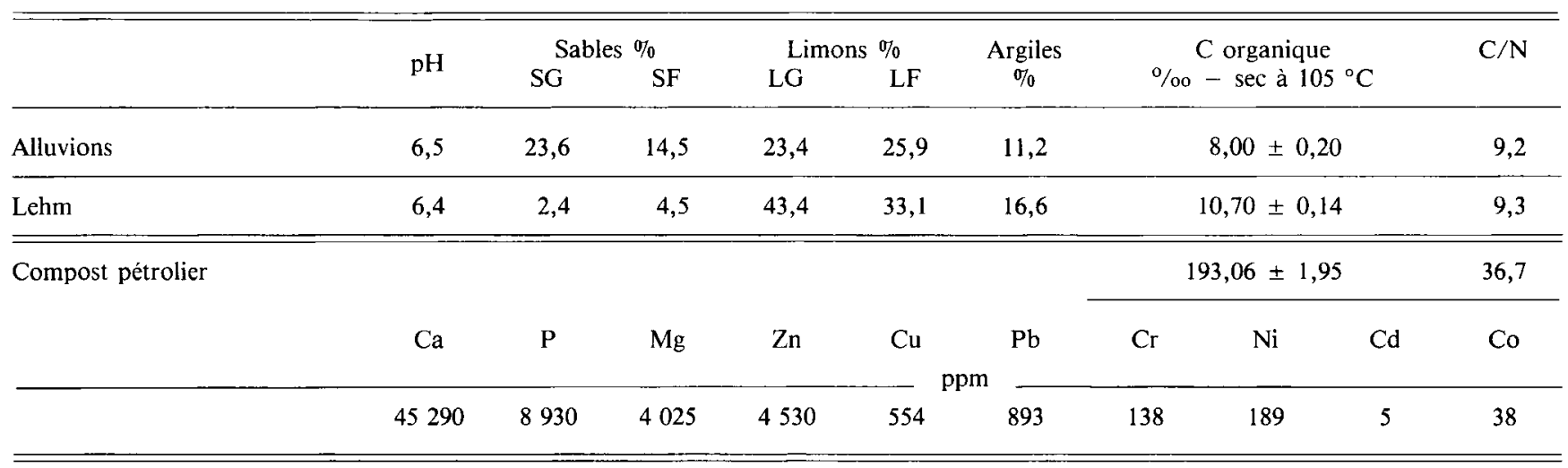


TABLEAU 2

Teneurs en carbone organique des mélanges et contribution du $C$ organique du compost au carbone total du mélange. Organic carbon contents of the mixtures and share of manure carbon in total carbon.

\begin{tabular}{lccc}
\hline \hline & $\begin{array}{c}\text { Taux du C org. } \\
\%-\sec \text { à } 105{ }^{\circ} \mathrm{C}\end{array}$ & $\begin{array}{c}\mathrm{C} \text { org. du compost } \\
\% \text { du C total }\end{array}$ & $\begin{array}{c}\mathrm{C} \text { du compost } \\
\mathrm{C} \text { initial des sols }\end{array}$ \\
\hline $\begin{array}{l}\text { Alluvions } \\
+ \text { Compost } \\
\begin{array}{l}\text { Lehm } \\
+ \text { Compost }\end{array}\end{array}$ & $\begin{array}{c}10,45 \pm 0,23 \\
(* 1)\end{array}$ & $24,45 \pm 0,20$ & 32,5 \\
\hline \\
\hline \hline
\end{tabular}

* Le dosage des mélanges a donné les résultats suivants :

(1) : $10,12 \pm 0,17$ et (2) : $12,72 \pm 0,17 \mathrm{mg} / \mathrm{g}$ de mélange sec à $105^{\circ} \mathrm{C}$.

kilogrammes ont été obtenus par quartage. Certains lots sont affectés aux analyses immédiates, d'autres sont disposés dans des cuvettes pour subir une période de 9 mois d'incubation dans une enceinte climatisée maintenue à $20^{\circ} \mathrm{C}$. Les échantillons de sols non enrichis en compost subissent, comme témoins, le même traitement. Les incubations ont été faites sur des échantillons maintenus à l'humidité équivalente $(\mathrm{pF}=3)^{*}$.

Le $\mathrm{CO}_{2}$ produit par la minéralisation des matières organiques des sols et du compost est collecté périodiquement en disposant une coupelle de soude diluée à la surface du sol et en plaçant le tout dans un sac de plastique fermé hermétiquement. Le $\mathrm{CO}_{2}$ dégagé en 24 heures est mesuré par titrage de la soude carbonatée. Les résultats présentés sur la figure 1 constituent la moyenne de 4 répétitions et sont limités aux 163 premiers jours de l'épreuve.

\section{Méthodes d'analyses des échantillons}

\section{Datations ${ }^{14} \mathrm{C}$}

Les mesures de la radioactivité naturelle ${ }^{14} \mathrm{C}$ ont été faites selon les méthodes de GUILLET (1972), BALESDENT \& GUILLET (1982) sur le compost pétrolier et sur les sols enrichis ou non en compost, mais ayant subi l'incubation. Par l'activité spécifique du carbone, on déduit la part prise par le carbone du résidu pétrolier dans le carbone total du mélange après incubation. La part $(\mathrm{x})$, ou le pourcentage $(100 \mathrm{x})$, de carbone du résidu pétrolier est calculée à partir de l'activité spécifique du compost pétrolier $(\mathrm{A} \pm \mathrm{a}$ ) et de celles du sol seul $(\mathrm{B} \pm \mathrm{b})$ et du mélange $(\mathrm{C} \pm \mathrm{c})$, selon la formule :

$$
\mathrm{xA}+(1-\mathrm{x}) \mathrm{B}=\mathrm{C} \text {. }
$$

En introduisant l'incertitude (écart-type $1 \sigma$ ) sur le pourcentage, on résoud,

$$
\begin{aligned}
\mathrm{x} \pm \Delta \mathrm{x}= & \frac{\mathrm{B}-\mathrm{C}}{\mathrm{B}-\mathrm{A}} \pm \\
& \pm \frac{\mathrm{a}(\mathrm{B}-\mathrm{C})+\mathrm{b}(\mathrm{C}-\mathrm{A})+\mathrm{c}(\mathrm{B}-\mathrm{A})}{(\mathrm{B}-\mathrm{A})^{2}}
\end{aligned}
$$

\footnotetext{
* On rajoute deux fois par semaine la quantité d'eau perdue par évaporation en ramenant au poids initial.
}

\section{Fractionnement des matières organiques}

Le fractionnement a été réalisé selon la méthode de BRUCKERT \& GAIFFE, 1983. Les fractions humiques $\mathrm{CH}_{5}, \mathrm{CH}_{10}, \mathrm{CH}_{12}$ sont extraites respectivement et successivement par la dithionite de sodium $(6 \mathrm{p} .100)$ à $\mathrm{pH} 5$, le pyrophosphate de sodium $0,1 \mathrm{M}$ à $\mathrm{pH} 10$, la soude $0,1 \mathrm{M}$ à $\mathrm{pH} 12$. Une dissolution complète des «ciments humiques » étant obtenue, les fractions argileuses COA et les débris végétaux fins et grossiers (DVF et DVG) sont séparés par fractionnement granulométrique à 2 et $50 \mu$. La fraction organo-argilique COA de nature peptidique (BRUCKERT \& KILBERTUS, 1980) est encore contaminée de débris hérités très fins.

Les dosages du carbone organique des échantillons solides ont été faits au Carmhograph WOSTOFF $\mathrm{n}^{\circ} 8$, ceux des échantillons liquides à l'analyseur $A S T R O$ (mod. 1859).

\section{Mesures des propriétés physiques et hydriques}

La stabilité structurale est déterminée selon le test de HENIN et al. (1969). Le test consiste à évaluer, par pesée, le pourcentage d'agrégats restant stables lorsque le sol est traité à l'eau, l'alcool et au benzène. Un indice de stabilité structurale (S) est calculé selon la formule de HENIN.

Le test de percolation mesure, selon la loi de DARCY, la perméabilité d'un échantillon de sol remanié. L'indice $K$ exprime la hauteur d'eau (en $\mathrm{cm}$ ), qui a percolé en 1 heure sous charge constante, à travers une colonne de sol de hauteur et section données.

Les limites d'ATTERBERG définissent les teneurs en eau caractéristiques de certains changement d'état ; la limite de plasticité (LP) sépare le domaine solide du domaine plastique, la limite de liquidité (LL) sépare ce dernier du domaine liquide.

Les tests de compactage ont permis de dresser les courbes de tassement Proctor qui illustrent la variation de densité sèche qs d'un sol, soumis à une énergie de compactage donnée, en fonction de l'humidité pondérale de l'échantillon.

Les relations potentiel de l'eau-humidité ont été précisées à partir des teneurs en eau des échantillons, déterminées par pression à des potentiels $(\mathrm{pF})$ de 2,5; 2,$7 ; 3,0 ; 4,0$ et 4,2 (presse à membrane de Richards, extraction par plaque de porcelaine). 


\section{RÉSULTATS}

\section{A. Biodégradation du compost pétrolier}

\section{Courbes de minéralisation en $\mathrm{CO}_{2}$ et quantités de matières minéralisées}

Les courbes (fig. 1) présentent les quantités de carbone minéralisé sous forme de $\mathrm{CO}_{2}$ par kilogramme de sol sec pendant $24 \mathrm{~h}$ aux dates indiquées. Elles présentent l'allure décroissante classique indiquant qu'après une minéralisation initiale importante des substances organiques (coup de fouet ou " flush »), les minéralisations quotidiennes fluctuent peu ensuite, à partir du $50^{\mathrm{e}}$ jour environ.
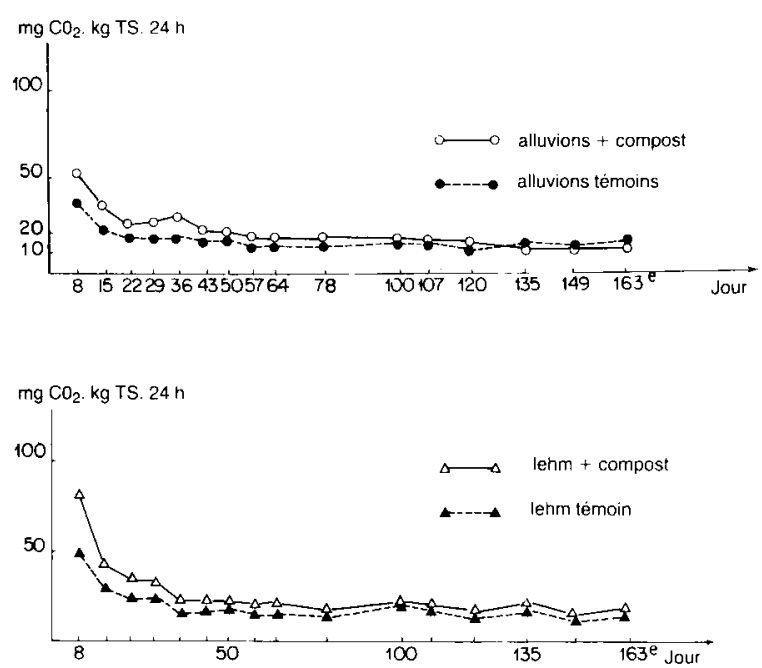

Figure 1

Quantités de $\mathrm{CO}_{2}$ dégagées pendant $24 \mathrm{~h}$ par $\mathrm{kg}$ de sol sec. Amounts of $\mathrm{CO}_{2}$ evolved in $24 \mathrm{~h}$ per $\mathrm{kg}$ dry soil.

Lors du «flush » biologique des premiers jours, on note que le lehm minéralise une plus grande quantité de matière organique que les alluvions : ces observations confirment les résultats obtenus par H. BOUSLAH (1982) qui notait une plus forte activité biologique dans le lehm.

La comparaison entre les courbes des sols amendés et celles des sols témoins montre qu'il y a eu minéralisation du compost pétrolier. Il est possible d'évaluer les quantités totales du carbone dégagé sous forme de
$\mathrm{CO}_{2}$ en calculant les valeurs quotidiennes par la méthode de l'interpolation linéaire entre deux observations consécutives. La somme de toutes les valeurs journalières, résultant de l'analyse ou du calcul, donne une évaluation de la quantité totale de $\mathrm{CO}_{2}$ produit.

Les résultats sont reportés dans le tableau 3 et comportent les évaluations établies du $5^{\mathrm{e}}$ au $47^{\mathrm{e}}$ jour et du $48^{\mathrm{e}}$ au $200^{\mathrm{e}}$ jour.

TABLEAU 3

Quantités de carbone minéralisé sous forme de $\mathrm{CO}_{2}$ (mg par $\mathrm{kg}$ de sol sec).

Amounts of carbon evolved as $\mathrm{CO}_{2}$ ( $\mathrm{mg}$ per $\mathrm{kg}$ dry soil).

\begin{tabular}{rcccc}
\hline & Alluvions & $\begin{array}{c}\text { Alluvions } \\
+ \text { Compost }\end{array}$ & Lehm & $\begin{array}{c}\text { Lehm } \\
+ \text { Compost }\end{array}$ \\
\hline $5^{\mathrm{e}}$ au $47^{\mathrm{e}} \mathrm{j}$ & 210 & 307 & 273 & 410 \\
$48^{\mathrm{e}}$ au $200^{\mathrm{e}} \mathrm{j}$ & 595 & 620 & 643 & 782 \\
Total & 805 & 927 & 916 & 1192 \\
\hline
\end{tabular}

Si l'on suppose que pendant l'incubation l'apport de compost pétrolier n'a causé ni effet stimulant (priming effect) ni effet dépressif sur la minéralisation endogène des sols, on peut estimer la quantité de $\mathrm{CO}_{2}$ produit par minéralisation du compost. Ainsi on admettra que la différence de $\mathrm{CO}_{2}$ dégagé, notée entre les échantillons témoins et les sols enrichis en compost est attribuable à la minéralisation des substances organiques du compost pétrolier. Ainsi, pour les alluvions, $122 \mathrm{mg}$ de carbone et pour le lehm $276 \mathrm{mg}$ proviendraient de la minéralisation de ces produits contenus dans un kilogramme du mélange sec.

A partir des données du tableau 2, précisant les taux de carbone organique des mélanges et le pourcentage pris dans ces mélanges par le carbone du compost pétrolier, on peut établir des taux de minéralisation. Ces taux sont obtenus par le rapport des quantités minéralisées sur les quantités initiales de carbone attribuables aux matières organiques endogènes des sols, ou exogènes du compost.

Les résultats du tableau 4 montrent qu'en 200 jours d'incubation, le compost pétrolier est minéralisé à des taux différents selon le sol avec lequel il est mélangé. En présence du lehm, le taux de minéralisation est plus du double de celui observé avec les alluvions (10,8 p. 100 contre 4,7 p. 100). Par contre, la miné-

TABLEAU 4

Taux de minéralisation du compost pétrolier et des matières organiques des sols après 200 jours d'incubation. (*exprimé en g par kg de sol sec.)

Mineralization rates of the fuel manure and of the soil organic matter after incubation for 200 days (in $\mathrm{g}$ per $\mathrm{kg}$ dry soil).

\begin{tabular}{lcccccc}
\hline \hline & Compost pétrolier & & \multicolumn{2}{c}{ Matière organique des sols } \\
\hline Alluvions + Compost & $\begin{array}{c}\text { Carbone } \\
\text { incorporé } \\
\text { au sol } \\
(\mathrm{en} \mathrm{g})\left(^{*}\right)\end{array}$ & $\begin{array}{c}\text { Carbone } \\
\text { dégagé sous } \\
\text { forme de CO } \\
(\mathrm{en} \mathrm{g})\left(^{*}\right)\end{array}$ & $\begin{array}{c}\text { Taux de } \\
\text { minéralisation }\end{array}$ & $\begin{array}{c}\text { Carbone } \\
\text { du sol }\end{array}$ & $\begin{array}{c}\text { Carbone } \\
\text { dégagé sous } \\
\text { forme de CO } \\
(\times 100)\end{array}$ & $\begin{array}{c}\text { Taux de } \\
\text { minéralisation } \\
(\mathrm{en} \mathrm{g})\left(^{*}\right)\end{array}$ \\
\hline Lehm + Compost & 2,555 & 0,122 & 4,7 & 7,895 & 0,806 & 10,2 \\
\hline \hline
\end{tabular}


ralisation endogène des sols s'effectuerait à des taux comparables (10,2 p. 100 dans les alluvions; 8,7 p. 100 pour le lehm). Ces valeurs assez élevées peuvent s'expliquer par la technique d'incubation faisant alterner dessiccation modérée et réhumectation et la durée de l'expérience.

Dans le test mettant en présence les alluvions et le compost, le taux de minéralisation plus élevé des matières organiques du sol conduit à penser que la part du compost en fin d'incubation se trouve être relevée. Un calcul simple $\left(^{*}\right)$ établit la contribution du carbone du compost pétrolier, initialement fixé à 24,45 p. 100 (tabl. 2) à 25,5 p. 100 en fin d'expérience. Pour ce qui concerne le mélange avec le lehm, comme les taux de minéralisation des deux partenaires sont voisins, la contribution du carbone du compost se trouve peu modifiée (19 p. 100 contre 19,4 p. 100 initialement, cf. tabl. 2).

\section{Activité spécifique ${ }^{14} \mathrm{C}$ du carbone organique}

L'activité ${ }^{14} \mathrm{C}$ du compost pétrolier a été mesurée et a donné une activité spécifique de 0,239 $\pm 0,089$ coup/ mn par gramme de carbone. C'est une valeur faible, mais mesurable qui rend compte du mélange de la boue de raffinerie, produit fossile d'activité nulle, avec de la paille qui aurait une activité spécifique voisine de 13,70 $\pm 0,40$ correspondant aux mesures de l'activité du $\mathrm{CO}_{2}$ atmosphérique des années 1980-1982, période pendant laquelle eut lieu le compostage. Dans ces conditions, un calcul simple indique que le carbone organique de la paille représente $1,75 \pm 0,05 \mathrm{p} .100 \mathrm{du}$ carbone du compost.

Comme il n'y a pas eu d'incubation du compost pétrolier seul, nous avons admis pour valeur (A) de la formule (2) son activité spécifique mesurée, soit de $0,239 \pm 0,089 \mathrm{coup} / \mathrm{mn} / \mathrm{g}$ de carbone. Ainsi, nous admettons implicitement que l'activité spécifique n'a pas varié au cours de l'incubation, ce qui est incontestablement abusif, car il est probable que la paille se soit minéralisée préférentiellement aux produits pétroliers et qu'ainsi elle participe à l'élévation des taux de minéralisation du compost pétrolier.

La contribution du carbone du compost pétrolier évaluée en fin d'expérience (tabl. 5) est assortie d'un écart-type qui limite la portée des valeurs indiquées. Il est certain que la mesure de l'activité spécifique du $\mathrm{CO}_{2}$ aurait donné des résultats plus précis que la méthode utilisée consistant à mesurer celle des matières organiques des sols et des mélanges. Néanmoins, les chiffres confirment les données précédentes.

En effet, pour les alluvions, la contribution du carbone du compost est augmentée en fin d'expérience $(25,47$ p. 100 contre 24,45 p. 100 initialement, cf. tabl. 2) et est évaluée de la même façon par le bilan des masses de $\mathrm{CO}_{2}$ que par les activités spécifiques résiduelles (respectivement 25,5 p. 100 et 25,47 p. 100). Ceci confirme bien que dans son mélange avec les alluvions, le compost s'est minéralisé à un taux inférieur à celui des matières organiques du sol. Par contre, pour le lehm, la contribution demeure inchangée $(19,42$ p. 100 contre 19,4 p. 100 du tabl. 2) indiquant de toute évidence que le compost et les matières organi-

(*) Les valeurs du tableau 4 donnent pour les alluvions : $(2,555-0,122) /((2,555-0,122)+(7,895-0,806))$. ques du sol se sont minéralisées selon des taux identiques ou voisins. Il y a là confirmation des valeurs du tableau 4.

Ainsi dans les deux tests, les mesures de l'activité spécifique $\mathrm{du}{ }^{14} \mathrm{C}$ naturel viennent corroborer les indications données par les mesures du $\mathrm{CO}_{2}$ dégagé par minéralisation et authentifient l'évaluation des taux de minéralisation. En outre, elles permettent de penser que le compost n'a exercé sur la minéralisation des matières organiques des sols ni effet stimulant ni effet dépressif significatifs.

TABLEAU 5

Activité spécifique ${ }^{14} \mathrm{C}$ et part du carbone du compost pétrolier restant dans les échantillons en fin d'expérience.

Specific activity of natural radiocarbon and share of the carbon of the fuel manure remaining in samples at the end of the experiment.

\begin{tabular}{|c|c|c|}
\hline & $\begin{array}{l}\text { Activité spécifique } \\
\text { (coup } / \mathrm{mn} / \mathrm{g} \\
\text { de carbone) }\end{array}$ & $\begin{array}{l}\text { Contribution du } \\
\text { carbone du compost } \\
\text { pétrolier en } \% \\
\text { du carbone total } \\
\text { en fin d'incubation }\end{array}$ \\
\hline Alluvions Témoin & $11,566 \pm 0,057$ & \\
\hline Alluvions + Compost & $8,680 \pm 0,050$ & $25,47 \pm 1,01$ \\
\hline Lehm Témoin & $10,781 \pm 0,055$ & \\
\hline Lehm + Compost & $8,733 \pm 0,051$ & $19,42 \pm 1,06$ \\
\hline
\end{tabular}

\section{B. Fractionnement de la matière organique}

La validité du fractionnement est attestée par la reproductibilité des répétitions et par la faible valeur des écarts relatifs entre somme de fraction et prise d'essai : la moyenne des valeurs absolues de ces écarts est en effet de 2,2 p. 100. Les résultats sont exprimés en valeur absolue, c'est-à-dire en $\mathrm{mg}$ de carbone issu d'un même poids d'échantillon fixé arbitrairement à $15 \mathrm{~g}$, parce que c'est l'un des moyens de localiser le compost dans l'une ou l'autre des fractions organiques et de déceler les transferts d'un compartiment à l'autre.

L'expression par contre des résultats en valeur relative (C p. 100 du C de l'échantillon) ne permet pas de donner une interprétation juste des faits. Pour s'en convaincre, voici la distribution des fractions contenues dans deux échantillons :

\begin{tabular}{llllllll}
\hline \hline & \multicolumn{6}{c}{ Fractions humiques } & $\begin{array}{c}\text { Frac- } \\
\text { tion } \\
\text { organo- } \\
\text { argi- } \\
\text { lique }\end{array}$ \\
& $\mathrm{CH}_{5}$ & $\mathrm{CH}_{10}$ & $\mathrm{CH}_{12}$ & $\mathrm{COA}$ & DVG & DVF \\
\hline $\begin{array}{l}\text { Lehm à 9 mois } \\
\text { Lehm + compost } \\
\text { à 9 mois }\end{array}$ & 30,5 & 24,3 & 13,1 & 21,1 & 5,3 & 5,7 \\
\hline- & 25,2 & 31,4 & 8,8 & 22,8 & 7,5 & 4,4 \\
\hline
\end{tabular}

La distribution paraît la même, alors qu'elle est très différente en réalité comme le montrent les chiffres en valeur absolue donnée plus loin dans le tableau 6 . 
TABLEAU 6

Répartition des matières organiques dans les fractions au départ et en fin d'expérience. (Quantités de carbone en $\mathrm{mg}$ dans une prise d'essai de $15 \mathrm{~g}$ de sol ou mélange.)

Distribution into fractions of organic matter at the beginning and at the end of the experiment ( $C$ in $m g$ in samples of $15 \mathrm{~g}$ of soil or mixture).

\begin{tabular}{|c|c|c|c|c|c|c|c|}
\hline \multicolumn{2}{|c|}{ Fractions } & $\mathrm{CH}_{5}$ & $\mathrm{CH}_{10}$ & $\mathrm{CH}_{12}$ & $\mathrm{COA}$ & DVG & DVF \\
\hline \multicolumn{8}{|c|}{ Traitements au départ de l'expérience } \\
\hline $\begin{array}{l}\text { Lehm Témoin } \\
\text { Lehm + Compost }\end{array}$ & $\begin{array}{l}0 \text { jour } \\
0 \text { jour }\end{array}$ & $\begin{array}{l}60,4 \\
75,7^{* *}\end{array}$ & $\begin{array}{l}21,4 \\
22,3\end{array}$ & $\begin{array}{l}30,3 \\
19,1\end{array}$ & $\begin{array}{l}15,3 \\
49,5^{* *}\end{array}$ & $\begin{array}{l}10,5 \\
13,9\end{array}$ & $\begin{array}{l}20,3 \\
10,3\end{array}$ \\
\hline $\begin{array}{l}\text { Alluvions Témoin } \\
\text { Alluvions }+ \text { Compost }\end{array}$ & $\begin{array}{l}0 \text { jour } \\
0 \text { jour }\end{array}$ & $\begin{array}{l}52,4 \\
72,0^{* *}\end{array}$ & $\begin{array}{l}12,0 \\
19,8^{* *}\end{array}$ & $\begin{array}{l}23,9 \\
21,2\end{array}$ & $\begin{array}{c}6,9 \\
25,9^{* *}\end{array}$ & $\begin{array}{l}13,6 \\
12,5\end{array}$ & $\begin{array}{r}10,1 \\
8,0\end{array}$ \\
\hline \multicolumn{8}{|c|}{ Traitements en fin d'expérience } \\
\hline $\begin{array}{l}\text { Lehm Témoin } \\
\text { Lehm + Compost }\end{array}$ & $\begin{array}{l}9 \text { mois } \\
9 \text { mois }\end{array}$ & $\begin{array}{l}41,2 \\
40,9\end{array}$ & $\begin{array}{l}32,8 \\
51,3^{* *}\end{array}$ & $\begin{array}{l}17,5 \\
14,3\end{array}$ & $\begin{array}{l}28,5 \\
37,2^{* *}\end{array}$ & $\begin{array}{r}7,2 \\
12,3\end{array}$ & $\begin{array}{l}7,8 \\
7,2\end{array}$ \\
\hline $\begin{array}{l}\text { Alluvions Témoin } \\
\text { Alluvions }+ \text { Compost }\end{array}$ & $\begin{array}{l}9 \text { mois } \\
9 \text { mois }\end{array}$ & $\begin{array}{l}49,2 \\
49,4\end{array}$ & $\begin{array}{c}6,6 \\
15,0^{* *}\end{array}$ & $\begin{array}{c}6,9 \\
20,8^{* *}\end{array}$ & $\begin{array}{l}24,0 \\
43,2^{* *}\end{array}$ & $\begin{array}{l}12,6 \\
11,4\end{array}$ & $\begin{array}{l}5,7 \\
4,2\end{array}$ \\
\hline
\end{tabular}

** Fraction enrichie par rapport au sol témoin.

\section{Localisation de la matière organique issue du compost}

Au départ de l'expérience (tabl. 6), la comparaison des témoins et des échantillons amendés montre que le compost pétrolier se retrouve surtout dans les fractions argiliques (COA) et extractibles à $\mathrm{pH} 5$ par la dithionite $\left(\mathrm{CH}_{5}\right)$. La baisse des fractions $\mathrm{CH}_{12}$ et DVF proviendrait d'un effet physique instantané qui s'exercerait plus sur l'échantillon limoneux (lehm) que sur l'échantillon sableux (alluvions).

En fin d'expérience, le compost se localise encore dans la fraction argilique, mais aussi dans les fractions de composés humiques $\mathrm{CH}_{10}$ (lehm), $\mathrm{CH}_{10}$ et $\mathrm{CH}_{12}$ (alluvions). Par contre, la fraction $\mathrm{CH}_{5}$ ne renferme apparemment plus de compost.

\section{Analyse des transferts d'un compartiment organi- nique à l'autre}

L'évolution de la matière organique endogène entre le début et la fin de l'expérience est donnée par les chiffres du tableau 7. La biodégradation qui entraîne, on le sait, une disparition des matières organiques (minéralisation), mais aussi des formations de métabolites (humification), provoque à la fois des diminutions de certaines fractions et des augmentations d'autres fractions : il apparaît clairement que des transferts d'un compartiment à l'autre se produisent au cours de l'incubation. On constate une baisse des débris végétaux et des composés extractibles à $\mathrm{pH} 5$ et à $\mathrm{pH} 12$. On assiste corrélativement à un accroissement de la fraction argilique. L'évolution de la fraction extractible $\mathrm{CH}_{10}$ diffère selon chacune des terres étudiées : cette fraction augmente dans le lehm et diminue dans les alluvions. Les substances extraites à $\mathrm{pH} 10$ étant des composés spécifiquement humiques, il semble que les transformations vers une humification soient plus avancées dans le lehm que dans les alluvions.

L'évolution du compost pétrolier en mélange avec la matière organique des sols est donnée par les chiffres du tableau 7. On constate que les petites molécules extraites à $\mathrm{pH} 5$ ont fortement diminué dans les deux sols étudiés. Cette baisse, due aux processus de minéralisation et d'humification s'est faite au profit de com-

TABLEAU 7

Effet de l'incubation sur la matière organique : comparaison des fractions au temps 0 et 9 mois. (Quantité de carbone en mg dans une prise d'essai de $15 \mathrm{~g}$ de sol ou mélange.)

Change of organic fractions during the experiment ( $C$ in $\mathrm{mg}$ per $15 \mathrm{~g}$ of soil or mixture).

\begin{tabular}{|c|c|c|c|c|c|c|c|}
\hline \multicolumn{2}{|c|}{ Fractions } & \multirow[t]{2}{*}{$\mathrm{CH}_{5}$} & \multirow[t]{2}{*}{$\mathrm{CH}_{10}$} & \multirow[t]{2}{*}{$\mathrm{CH}_{12}$} & \multirow[t]{2}{*}{$\mathrm{COA}$} & \multirow[t]{2}{*}{ DVG } & \multirow[t]{2}{*}{ DVF } \\
\hline Traitements Témoins & & & & & & & \\
\hline $\begin{array}{l}\text { Lehm Témoin } \\
\text { Lehm Témoin }\end{array}$ & $\begin{array}{l}0 \text { jour } \\
9 \text { mois }\end{array}$ & $\begin{array}{l}60,4 \\
41,2\end{array}$ & $\begin{array}{l}21,4 \\
32,8\end{array}$ & $\begin{array}{l}30,3 \\
17,5\end{array}$ & $\begin{array}{l}15,3 \\
28,5^{* *}\end{array}$ & $\begin{array}{r}10,5 \\
7,2\end{array}$ & $\begin{array}{r}20,3 \\
7,8\end{array}$ \\
\hline $\begin{array}{l}\text { Alluvions Témoin } \\
\text { Alluvions Témoin }\end{array}$ & $\begin{array}{l}0 \text { jour } \\
9 \text { mois }\end{array}$ & $\begin{array}{l}52,4 \\
49,2\end{array}$ & $\begin{array}{r}12,0 \\
6,6\end{array}$ & $\begin{array}{r}23,9 \\
6,9\end{array}$ & $\begin{array}{c}6,9 \\
24,0^{* *}\end{array}$ & $\begin{array}{l}13,6 \\
12,6\end{array}$ & $\begin{array}{r}10,1 \\
5,7\end{array}$ \\
\hline \multicolumn{8}{|c|}{ Traitements enrichis en compost pétrolier $40 \mathrm{t} / \mathrm{ha}$} \\
\hline $\begin{array}{l}\text { Lehm }+ \text { Compost } \\
\text { Lehm }+ \text { Compost }\end{array}$ & $\begin{array}{l}0 \text { jour } \\
9 \text { mois }\end{array}$ & $\begin{array}{l}75,7 \\
40,9\end{array}$ & $\begin{array}{l}22,3 \\
51,3 * *\end{array}$ & $\begin{array}{l}19,1 \\
14,3\end{array}$ & $\begin{array}{l}49,5 \\
37,2\end{array}$ & $\begin{array}{l}13,9 \\
12,3\end{array}$ & $\begin{array}{r}10,3 \\
7,2\end{array}$ \\
\hline $\begin{array}{l}\text { Alluvions + Compost } \\
\text { Alluvions + Compost }\end{array}$ & $\begin{array}{l}0 \text { jour } \\
9 \text { mois }\end{array}$ & $\begin{array}{l}72,0 \\
49,4\end{array}$ & $\begin{array}{l}19,8 \\
15,0\end{array}$ & $\begin{array}{l}21,2 \\
20,8\end{array}$ & $\begin{array}{l}25,9 \\
43,2^{* *}\end{array}$ & $\begin{array}{l}12,5 \\
11,4\end{array}$ & $\begin{array}{l}8,0 \\
4,2\end{array}$ \\
\hline
\end{tabular}

** Fraction enrichie par rapport au temps $t_{0}$. 
posés spécifiquement humiques dans le lehm et au profit de matière organique liée aux argiles et de nature probablement peptidique dans les alluvions.

Les décalages observés dans les transformations des matières organiques du lehm et des alluvions sont à mettre en relation avec la composition texturale et les propriétés physico-chimiques différentes des deux terres.

\section{Les effets du compost pétrolier sur les propriétés physiques des sols}

\section{Stabilité structurale}

Les taux d'agrégats stables après l'action de l'alcool, eau et benzène, sont consignés sur le tableau 8. Dans les deux sols il faut noter le net accroissement des taux d'agrégats stables au benzène (agb) pour les échantillons ayant reçu du compost pétrolier. Celui-ci améliore donc la stabilité structurale des sols ainsi que le démontrent les valeurs de l'indice de HENIN (log $10 \mathrm{~S}$ ) qui, rappelons-le, varie en raison inverse de la stabilité structurale.

Ces résultats confirment l'effet primordial du compost pétrolier sur la mouillabilité des sols déjà mis en évidence lors du premier travail (H. BOUSLAH, 1982). Ils confirment également l'absence d'effet sur le coefficient de perméabilité $K$.

\section{Domaine de plasticité}

Le comportement mécanique d'un sol en fonction de l'humidité peut se caractériser globalement par les limites d'ATTERBERG : limite de plasticité (LP) et limite de liquidité (LL). On définit également une limite d'adhésivité (LA) si l'on veut connaître le taux d'humidité pour lequel la terre commence à adhérer au métal d'un outil. De nombreuses études ont montré que le taux de la matière organique joue un rôle sur les limites de liquidité et de plasticité dont il élève respectivement la valeur, mais sans que ces variations affectent l'indice de plasticité (IP = LL - LP) (METTAUER et al., 1983).

Ces considérations et l'objectif de valorisation agronomique nous ont conduits à tester les éventuels effets du résidu sur le comportement mécanique des sols. Le tableau 9 résume les valeurs obtenues sur des échantillons moyens respectifs des sols enrichis et des témoins après les 9 mois d'incubation.

A cause de sa texture plus fine les limites de plasticité, adhésivité et liquidité sont atteintes chez le lehm pour des teneurs en eaux supérieures à celles du sol alluvial. Mais il faut convenir que l'apport du compost pétrolier est sans effet sur ces paramètres physiques.

\section{Sensibilité au tassement}

Si l'on compacte d'une manière dynamique des échantillons de sols d'humidité croissante (test PROCTOR) on obtient une courbe de tassement qui se caractérise par la variation de la densité sèche qs du sol.

Au-delà d'une humidité critique Wc, fonction de la teneur en argile, s'amorce un tassement important (forte pente de la courbe) pour atteindre une valeur maximale $(\mathrm{Wm})$. Ce tassement rapide est lié aux propriétés "lubrifiantes" de l'argile. Les travaux de FAURE et de GUÉRIF (1979) ont aussi montré que la matière organique possédait la propriété de diminuer la sensibilité au tassement des sols en réduisant le pouvoir lubrifiant des argiles (diminution importante de la pente de la courbe).

Ces diverses propriétés nous ont conduits à vérifier si les effets du compost pétrolier sont du même ordre de grandeur que ceux de la matière organique. La

TABLEAU 8

Taux d'agrégats stables en $\mathrm{g}$ pour $100 \mathrm{~g}$ de sol. Indice de HENIN et coefficient de perméabilité (log $10 K)$. Erreur $(1$ o) calculée sur 4 répétitions.

Rate of stable aggregates in $\mathrm{g}$ per $100 \mathrm{~g}$ of soils. HENIN index and permeability coefficient. Error $(1 \mathrm{\sigma})$ calculated on 4 replicates.

\begin{tabular}{lccccc}
\hline \hline & $\begin{array}{c}\text { Alcool } \\
\text { (aga) }\end{array}$ & $\begin{array}{c}\text { Agrégats } \\
\text { Eau } \\
\text { (age) }\end{array}$ & $\begin{array}{c}\text { Benzène } \\
\text { (agb) }\end{array}$ & $\log 10 \mathrm{~S}$ & $\log 10 \mathrm{~K}$ \\
\hline & $6,52 \pm 0,94$ & $2,88 \pm 0,23$ & $2,49 \pm 0,23$ & $1,42 \pm 0,03$ & $1,30 \pm 0,01$ \\
Alluvions (Témoin) & $5,76 \pm 0,82$ & $5,08 \pm 0,51$ & $14,07 \pm 2,34$ & $1,11 \pm 0,07$ & $1,30 \pm 0,01$ \\
Alluvions + Compost & $6,35 \pm 0,54$ & $2,64 \pm 0,08$ & $2,60 \pm 1,60$ & $1,75 \pm 0,10$ & $1,46 \pm 0,01$ \\
\hline Lehm (Témoin) & $10,88 \pm 1,30$ & $7,82 \pm 1,43$ & $19,53 \pm 4,08$ & $1,20 \pm 0,07$ & $1,50 \pm 0,04$ \\
Lehm + Compost & & & & \\
\hline
\end{tabular}

TABLEAU 9

Limites d'ATTERBERG. Indices de plasticité et limite d'adhésivité.

ATTERBERG consistency constants. Plasticity index and sticky point.

\begin{tabular}{lcccc}
\hline \hline & $\begin{array}{c}\text { Limite de liquidité } \\
\text { LL \% }\end{array}$ & $\begin{array}{c}\text { Limite de plasticité } \\
\text { LP \% }\end{array}$ & $\begin{array}{c}\text { Indice de plasticité } \\
\text { LL - LP \% }\end{array}$ & $\begin{array}{c}\text { Limite d'adhésivité } \\
\text { LA \% }\end{array}$ \\
\hline Alluvions Témoin & & & & 17,8 \\
Alluvions + Compost & 24,8 & 18,4 & 6,4 & 18,5 \\
Lehm Témoin & 24,5 & 20,5 & 4,0 & 23,5 \\
Lehm + Compost & 31,1 & 25,4 & 5,7 & 23,6 \\
\hline \hline
\end{tabular}




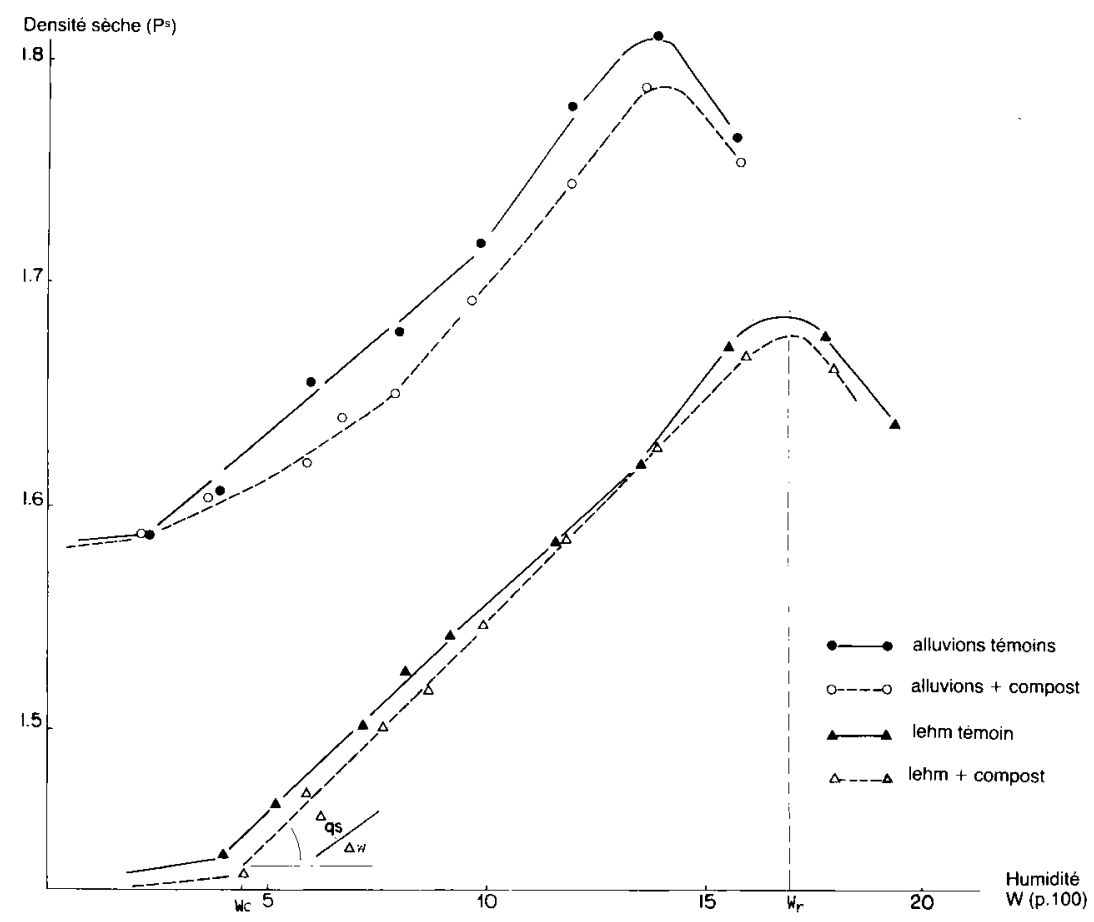

Figure 2

Courbes de compactage. PROCTOR.

Compaction curves. PROCTOR.

figure 2 représente les courbes de compactage réalisées sur les sols témoins et enrichis après 9 mois d'incubation. Leur interprétation conduit aux conclusions suivantes :

- les courbes de tassement des sols enrichis en compost ne se situent que très légèrement en dessous de celles des témoins correspondants. Si la translation peut être considérée comme significative pour le sol alluvial, elle est quasi nulle pour le lehm ;

- les pentes moyennes $\Delta \mathrm{qs} / \Delta \mathrm{W}$ des courbes de tassement des mélanges ne diffèrent nullement de celles des sols témoins. Cela signifie que le compost n'a pas eu d'effet sur le pouvoir lubrifiant de l'argile contrairement à celui de la matière organique endogène des sols ;

- l'effet du compost sur l'humidité critique Wc apparaît également négligeable.

En conclusion, le compost pétrolier n'agit pas sur le seuil de plasticité d'un sol à l'énergie de compactage mise en œuvre $(6 \mathrm{kgm})$ et n'exerce aucune influence amélioratrice sur la sensibilité au tassement des sols traités.

\section{Relations potentiel de l'eau ( $\psi)$ et taux d'humidité du sol}

Connaissant l'action bénéfique de la matière organique sur la dynamique de l'eau dans les sols, il apparaissait utile de tester aussi l'impact du compost pétrolier sur la capacité de rétention de l'eau d'un sol. Pour cela nous avons déterminé expérimentalement quelques points des courbes $(\psi(W))$ des sols témoins et enrichis.

Le tableau 10 résume ces données et montre qu'après 9 mois d'incubation, on ne constate aucune différence significative entre les humidités des sols témoins et celles des sols enrichis. L'effet du compost pétrolier sur les réserves utiles d'un sol est donc négligeable. Il faut toutefois signaler que l'un de nous, BOUSLAH (1982), a trouvé un effet positif“'significatif du compost sur la diminution de l'évaporation du sol amendé par rapport au sol témoin, résultat confirmé par les travaux de BARRES \& SAUTER (1984).

\section{CONCLUSION}

La minéralisation du compost pétrolier mis à incuber en présence de sols à texture limoneuse, a pu être

TABLEAU 10

Taux d'humidité (\%) à différents potentiels $(p F)$, moyennes de 4 répétitions, $(1,10)$ : coefficients de variation. Soil moisture rates (\%) at different matric potentials $(p F)$, mean of 4 replicates, (1.10): coefficient of variation.

\begin{tabular}{|c|c|c|c|c|c|}
\hline & $\mathrm{pF}=4,2$ & $\mathrm{pF}=4$ & $\mathrm{pF}=3$ & $\mathrm{pF}=2,7$ & $\mathrm{pF}=2,5$ \\
\hline $\begin{array}{l}\text { Alluvions Témoin } \\
\text { Alluvions }+ \text { Compost }\end{array}$ & $\begin{array}{l}5,1(0,25) \\
5,2(0,21)\end{array}$ & $\begin{array}{l}6,1(0,76) \\
6,1(0,24)\end{array}$ & $\begin{array}{l}16,8(1,22) \\
16,9(2,75)\end{array}$ & $\begin{array}{l}19,7(0,48) \\
19,3(1,45)\end{array}$ & $\begin{array}{l}20,3(1,10) \\
20,3(2,23)\end{array}$ \\
\hline $\begin{array}{l}\text { Lehm Témoin } \\
\text { Lehm }+ \text { Compost }\end{array}$ & $\begin{array}{l}7,2(0,41) \\
7,3(0,40)\end{array}$ & $\begin{array}{l}8,1(1,38) \\
8,3(1,90)\end{array}$ & $\begin{array}{l}21,0(0,97) \\
21,2(0,68)\end{array}$ & $\begin{array}{l}24,9(0,66) \\
25,0(0,40)\end{array}$ & $\begin{array}{l}26,5(0,21) \\
25,9(0,76)\end{array}$ \\
\hline
\end{tabular}


évaluée en mesurant le gaz carbonique dégagé au cours de l'incubation de 9 mois. Il semble que dans des conditions optimales d'humidité et de température, le taux de minéralisation du compost puisse approcher 5 p. 100 lors qu'il est apporté au sol alluvial et même atteindre 10 p. 100 en présence de lehm biologiquement plus actif.

Parallèlement à la biodégradation et la minéralisation, des transformations notables se sont produites au cours de l'incubation. Les produits pétroliers semblent avoir acquis des groupements fonctionnels qui favorisent leur solubilité dans les réactifs alcalins. Les procédés de fractionnement des matières organiques ont en effet montré qu'entre le début et la fin de l'expérience d'incubation on assiste à un transfert partiel du carbone de la fraction insoluble granulométriquement fine (fraction COA) vers la fraction soluble dans le pyrophosphate $\mathrm{Na}$ (fraction $\mathrm{CH}_{10}$ ). Des recherches sont en cours pour analyser de façon plus détaillée ces deux fractions.

Considéré comme amendement, le compost pétrolier, comme toute matière organique, améliore la stabi- lité structurale des sols grâce à un effet sur la mouillabilité. Mais là s'arrête son rôle bénéfique : il n'agit en effet ni sur la sensibilité au tassement, ni sur la plasticité, ni sur les réserves utiles en eau. Rappelons aussi que le compost pétrolier n'agit pratiquement pas sur la capacité d'échange des sols (BOUSLAH, 1982).

La valorisation agricole de ce compost doit être interprétée d'autant plus prudemment que les faibles effets bénéfiques peuvent être contrebalançés par des effets toxiques importants. Il faut en effet rappeler qu'à la suite de tests agronomiques de culture de RayGrass, BOUSLAH (1982) a montré qu'une perte de rendement évaluée à $15 \mathrm{p}$. 100 se produisait dès la dose de $20 \mathrm{t} / \mathrm{ha}$ et qu'un effet toxique notable apparaissait pour des apports de plus de $50 \mathrm{t} / \mathrm{ha}$.

\section{REMERCIEMENTS}

Ces recherches ont été subventionnées par le Ministère de l'Environnement.

Reçu le 3 octobre 1986 Accepté le 4 mars 1987.

\section{RÉFÉRENCES BIBLIOGRAPHIQUES}

Balesdent S., Guillet B., 1982. Les datations par le ${ }^{14} \mathrm{C}$ des matières organiques des sols. Contribution à l'étude de l'humification et du renouvellement des substances humiques. Science du Sal, 2, 93-112.

Barres M., Sauter M., 1984. Contribution au suivi sur terrains expérimentaux de la migration d'hydrocarbures dans la zone non saturée. Transferts vers la nappe. Rapport du B.R.G.M. (85 SGN 093 EAU), $27 \mathrm{p}$.

Bouslah H., 1982. Etude des sols de la bordure sous-vosgienne et des formations alluviales de la Thur (Alsace). Essai d'amélioration par apport de résidu pétrolier. Thèse de spécialité, Nancy, 120 p. + annexes.

Bruckert S., Gaiffe M., 1983. Essai de séparation des principales fractions organiques des sols. Bilan et caractérisation analytique. C. R. Acad. Sci., Paris, 297, SII, 627-630.

Bruckert S., Kilbertus G., 1980. Fractionnement et analyse des complexes organo-minéraux de sols bruns et de chernozems. Plant and Soil, 57, 271-295.

Carbonel J. P., Fourbet J. F., Fustec E., Gatellier C., Jacquesy R., 1982. In : Recovery of energy and material from residues and waste, 433-438. Ed. J. THOME-KozMiensky.
Gatellier C., 1983. Epandage de déchets pétroliers dans les sols. In : Protection des sols et devenir des déchets (Symposium à La Rochelle, p. 95-98) édité par Recherche Scientifique et Technique sur l'Environnement (1985).

Guérif J., Faure A., 1979. Rôle de la matière organique sur le comportement des sols au compactage. I. - Etude statistique. Ann. Agro., 30 (5), 387-399.

Guillet B., 1972. Datation des sols par le ${ }^{14} \mathrm{C}$ Naturel. I. - La méthode de datation en scintillation liquide. Bull. ENSAIA, Nancy, 14 (1), 117-122.

Hénin S., Gras R., Monnier G., 1969. Le Profil Cultural. L'état physique du sol et les conséquences agronomiques. Ed. : Masson, $332 \mathrm{p}$.

Mettauer H., Tual Y., Huck C., Trendel R., 1983. De la connaissance du comportement physique et mécanique des sols de l'Est de la France. Agron., 3 (2), 141-152. 